Article

\title{
Positive Energy Condition and Conservation Laws in Kantowski-Sachs Spacetime via Noether Symmetries
}

\author{
Sumaira Saleem Akhtar ${ }^{1}$, Tahir Hussain ${ }^{1} \mathbb{D}$ and Ashfaque H. Bokhari ${ }^{2, *} \mathbb{D}$ \\ 1 Department of Mathematics, University of Peshawar, Peshawar 25000, Khyber Pakhtunkhwa, Pakistan; \\ sumairamaths@gmail.com (S.S.A.); tahirhussain@uop.edu.pk (T.H.) \\ 2 Department of Mathematics and Statistics, King Fahd University of Petroleum and Minerals, Dhahran 31261, \\ Saudi Arabia \\ * Correspondence: abokhari@kfupm.edu.sa
}

Received: 16 November 2018; Accepted: 30 November 2018; Published: 4 December 2018

check for updates

\begin{abstract}
In this paper, we have investigated Noether symmetries of the Lagrangian of Kantowski-Sachs spacetime. The associated Lagrangian of the Kantowski-Sachs metric is used to derive the set of determining equations. Solving the determining equations for several values of the metric functions, it is observed that the Kantowski-Sachs spacetime admits the Noether algebra of dimensions 5, 6, 7, 8, 9, and 11. A comparison of the obtained Noether symmetries with Killing and homothetic vectors is also presented. With the help of Noether's theorem, we have presented the expressions for conservation laws corresponding to all Noether symmetries. It is observed that the positive energy condition is satisfied for most of the obtained metrics.
\end{abstract}

Keywords: Noether symmetries; conservation laws; Kantowski-Sachs metric

PACS: 04.20.Jb

\section{Introduction}

Einstein's field equations (EFEs), $G_{a b}=k T_{a b}$, are ten-tensor equations, which describe the gravitational effects produced by a given mass in a spacetime. In these equations, the stress-energy tensor $T_{a b}$ gives the distribution of energy and momentum, $k$ represents the gravitational constant, while $G_{a b}$ expresses the spacetime curvature and is known as the Einstein tensor. Moreover, the Einstein tensor $G_{a b}$ can be expressed as $G_{a b}=R_{a b}-\frac{R}{2} g_{a b}$, where $R_{a b}=$ the Ricci tensor, $g_{a b}=$ the metric tensor, and $R=$ the Ricci scalar.

The quantities $R_{a b}$ and $R$ appearing in the EFEs are built up from $g_{a b}$ and its partial derivatives. In this way, the EFEs form a system of partial differential equations. A Lorentz metric $g_{a b}$ is regarded as an exact solution of EFEs if it is obtained by solving the EFEs exactly in closed form and is conformable to a physically realistic $T_{a b}$. Finding the exact solutions of EFEs is not an easy task unless some simplifying assumptions are employed. Therefore, only a few physically meaningful solutions of these equations are found in the literature [1]. Among the approaches followed for obtaining the exact solutions of EFEs, the most popular is to use some symmetry restrictions on the tensor $g_{a b}$. These restrictions are mathematically expressed as $\mathcal{L}_{X} g_{a b}=0$, where $X$ is called a Killing vector $(\mathrm{KV})$ and $\mathcal{L}$ denotes the Lie derivative operator. It is well known that every KV corresponds to a conservation law. A large body of the literature is devoted to the investigation of KVs and corresponding conservation laws in spacetimes [1-3].

The symmetries of tensors other than the metric tensor are usually referred to as collineations. Some examples of collineations include curvature collineations $\left(\mathcal{L}_{X} R_{b c d}^{a}=0\right)$, Ricci collineations 
$\left(\mathcal{L}_{X} R_{a b}=0\right)$, and matter collineations $\left(\mathcal{L}_{X} T_{a b}=0\right)$. These collineations have also been thoroughly discussed [4-9].

Apart from the conventional symmetries defined above, the idea of another type of symmetry, known as Noether symmetry (NS), was given by Emmy Noether [10]. According to Noether's theorem, if the Lagrangian of a system admits a continuous symmetry, then this symmetry corresponds to a conservation law. Consequently, if a system remains unchanged under time translation and spacial translations and rotations, this theorem yields the conservation of energy and linear and angular momenta.

The study of NS is important due to the fact that it usually provides the additional conservation laws, not given by KVs. If the Killing and Noether algebras are denoted by $K(M)$ and $N(M)$, respectively, then $K(M) \subseteq N(M)$. The NS have also a close link with homothetic vectors (HVs). In fact, if $X$ is an $\mathrm{HV}$, that is $\mathcal{L}_{X} g_{a b}=2 \psi g_{a b} ; \psi$ being a constant, then $X+2 \psi u \partial_{u}$ is a Noether symmetry associated with $X$. Here, $u$ is the geodesics parameter of the world line of a point particle moving in a spacetime. Conversely, if $X+2 \psi u \partial_{u}$ is an NS, then $X$ is an $H V$, provided that $X$ is independent of $u$ [11]. A Noether symmetry that is neither a KV, nor associated with an $\mathrm{HV}$ is known as a proper Noether symmetry.

NS and their comparison with Killing and homothetic vectors have been studied for some well-known spacetimes. A comparative study of KVs and NS for the conformally-flat Friedmann metric was provided in [12], and it was concluded that this metric admits proper NS. Considering some specific spherically-symmetric metrics, Bokhari et al. [13] conjectured that the NS of the Lagrangian provide additional conservation laws. The NS of some other physically-important spacetimes were examined by different authors. Camci [14] studied the NS of geodesic motion for the geodesic Lagrangian of the metric of Gödel spacetimes. Camci and Yildirim [15] worked on the NS of the geodesic Lagrangian for some classes of pp-wave spacetimes. Hickman and Yazdan [11] studied NS in Bianchi type II spacetimes. They have shown that the Noether algebra of Bianchi type II spacetimes contain Killing, as well as homothetic vectors. Ali et al. [16-18] worked on the classification of different spacetimes via NS including static plane, static spherical, and static cylindrically-symmetric spacetimes. Jamil et al. $[19,20]$ worked on the complete classification of non-static plane and non-static spherically-symmetric spacetimes via NS. Paliathanasis et al. [21] established a relation between the Lie symmetries of the Klein-Gordon equation and conformal Killing vectors of the underlying geometry, where they also stated that the resulting Lie symmetries of the conformal algebra are also NS. Tsamparlis et al. [22] stated that for dynamical system whose equations of motion are of the form $\ddot{x}^{a}+\Gamma_{b c}^{a} \dot{x}^{b} \dot{x}^{c}+f\left(x^{a}\right)$; $f\left(x^{a}\right)$ being an arbitrary function of its argument, the computation of Lie and Noether symmetries reduces to the problem of finding the special projective collineations. Recently, the Bianchi type $\mathrm{V}$ and non-static plane symmetric spacetimes were completely classified via their NS [23,24].

It is important to mention here that the formulation of conservation laws may not require the Lagrangian of the system. As an example, one can see the work of Ma et al. [25], who have recently established a result giving the direct formulation of conservation laws for differential equations including the heat equation, Burgers' equation, and the Korteweg-de Vries (KdV) equation, regardless of the existence of a Lagrangian. The same authors also discussed the existence of lump solutions involving free parameters for some nonlinear partial differential equations that present Lie symmetries and that may generate associated conservation laws [26].

The Kantowski-Sachs spacetime describes a spatially-homogeneous and anisotropic universe model that admits an isometry group $G_{4}$ acting on homogeneous spacelike hypersurfaces. As the Kantowski-Sachs metric is of great interest and there is a great deal of information about this metric scattered throughout the literature, it would be useful to investigate the NS possessed by the Lagrangian of this metric and their relation with Killing and homothetic vectors.

For a physically-realistic spacetime, its energy density, represented by $T_{00}$, must be non-negative. This condition is usually referred to as the positive energy condition. The positive energy condition is important because if one allows both positive and negative energy regions, the empty vacuum may become 
unstable. In this paper, we classify the Lagrangian of the Kantowski-Sachs metric according to its NS and check the positive energy condition for the obtained models admitting different algebras of NS.

\section{Determining Equations}

The Kantowski-Sachs metric is given by [27]:

$$
d s^{2}=d t^{2}-\lambda^{2}(t) d r^{2}-v^{2}(t)\left(d \theta^{2}+\sin ^{2} \theta d \phi^{2}\right),
$$

where the functions $\lambda(t) \neq 0$ and $v(t) \neq 0$ depend on $t$ only. The minimum KVs admitted by the above metric are:

$$
\begin{aligned}
& X_{1}=\partial_{r}, \quad X_{2}=\partial_{\phi}, \\
& X_{3}=\sin \phi \partial_{\theta}+\cot \theta \cos \phi \partial_{\phi}, \\
& X_{4}=-\cos \phi \partial_{\theta}+\cot \theta \sin \phi \partial_{\phi} .
\end{aligned}
$$

One may use the EFEs with $k=1$ to get the following non-zero components of $T_{a b}$.

$$
\begin{aligned}
& T_{00}=\frac{1}{\lambda^{3} v^{2}}\left(v^{\prime 2} \lambda^{3}+2 \lambda^{2} v \lambda^{\prime} v^{\prime}+\lambda^{3}\right), \\
& T_{11}=-\frac{1}{v^{2}}\left(2 v \lambda^{2} v^{\prime \prime}+\lambda^{2} v^{\prime 2}+\lambda^{2}\right), \\
& T_{22}=-\frac{v}{\lambda^{3}}\left(\lambda^{3} v^{\prime \prime}+\lambda^{2} v^{\prime} \lambda^{\prime}+v \lambda^{2} \lambda^{\prime \prime}\right), \\
& T_{33}=\sin ^{2} \theta T_{22},
\end{aligned}
$$

where the primes on metric functions denote their derivatives with respect to $t$. The usual Lagrangian $L$ corresponding to the Kantowski-Sachs metric (1) is given by:

$$
L=\dot{t}^{2}-\lambda^{2}(t) \dot{r}^{2}-v^{2}(t)\left(\dot{\theta}^{2}+\sin ^{2} \theta \dot{\phi}^{2}\right),
$$

where the dot represents the derivative with respect to the parameter $u$ of the world line of a point particle moving in Kantowski-Sachs spacetime. The Lagrangian for this point particle, given in (4), represents the square of the Lagrangian for the action principle that leads to geodesics by minimizing the spacetime metric $\int d s=\sqrt{g_{a b} \frac{d x^{a}}{d u} \frac{d x^{b}}{d u}} d u$ [28]. It may be noted that the coefficients of the quadratic terms in $\frac{d x^{\mu}}{d u} \frac{d x^{v}}{d u}$ represent the components of the metric.

A vector field $X=\eta \frac{\partial}{\partial u}+X^{i} \frac{\partial}{\partial x_{i}}$ is called an NS if the Lagrangian of a physical system remains invariant under the action of $X$ such that the following condition holds:

$$
X^{(1)} L+L(D \eta)=D F
$$

where:

$$
X^{(1)}=X+X_{u}^{i} \frac{\partial}{\partial \dot{x}_{i}}
$$

is the first prolongation of $X$ such that $X_{u}^{i}=D X^{i}-\dot{x}_{i} D \eta$. Here, $D$ denotes the total differential operator, given by:

$$
D=\frac{\partial}{\partial u}+\dot{x}_{i} \frac{\partial}{\partial x_{i}} .
$$

Moreover, $\eta, X^{i}$, and $F$ all depend on the variables $\left(u, x_{i}\right)$, where $x_{i}=(t(u), r(u), \theta(u), \phi(u))$. The function $F$ is known as the gauge function. Once we find the NS and corresponding gauge function, we can use Noether's theorem to find the corresponding conservation laws in the following way: 


$$
I=\eta L+\left(X^{i}-\dot{x}_{i} \eta\right) \frac{\partial L}{\partial \dot{x}_{i}}-F .
$$

We use the Lagrangian given in Equation (4) in the equation defining the NS, that is Equation (5), to get the following set of 19 equations.

$$
\begin{aligned}
F_{, u}=\eta_{, \phi}=\eta_{, \theta}=\eta, r=\eta, t & =0, \\
2 X_{, t}^{0} & =\eta, u, \\
2 \lambda^{\prime} X^{0}+2 \lambda X_{, r}^{1} & =\lambda \eta, u, \\
2 v^{\prime} X^{0}+2 v X_{, \theta}^{2} & =v \eta, u \\
X_{, r}^{0}-\lambda^{2} X_{, t}^{1} & =0, \\
X_{, \theta}^{0}-v^{2} X_{, t}^{2} & =0, \\
X_{, \phi}^{0}-v^{2} \sin ^{2} \theta X_{, t}^{3} & =0, \\
\lambda^{2} X_{, \theta}^{1}+v^{2} X_{, r}^{2} & =0, \\
\lambda^{2} X_{, \phi}^{1}+v^{2} \sin ^{2} \theta X_{, r}^{3} & =0, \\
X_{, \phi}^{2}+\sin ^{2} \theta X_{, \theta}^{3} & =0, \\
2 v^{\prime} X^{0}+2 v \cot \theta X^{2}+2 v X_{, \phi}^{3} & =v \eta, u, \\
2 X_{, u}^{0}=F_{, t} & \\
2 \lambda^{2} X_{, u}^{1}=-F_{, r}, & \\
2 v^{2} X_{, u}^{2}=-F_{, \theta}, & \\
2 v^{2} \sin ^{2} \theta X_{, u}^{3}=-F_{, \phi} . &
\end{aligned}
$$

The solution of the above system of equations would give the values of the components of the vector field generating NS and the metric functions $\lambda$ and $v$. Consequently, the corresponding Kantowski-Sachs metrics may represent the exact solutions of EFEs. We omit writing the basic calculations and present the list of metrics, their NS, Lie algebras, and the corresponding conservation laws of all symmetries in the forthcoming sections. The bounds for the positive energy condition and the singularity of the Ricci scalar are also discussed for all the obtained metrics.

\section{Five Noether Symmetries}

The minimal set of NS admitted by the Lagrangian of the Kantowski-Sachs metric is:

$$
\begin{aligned}
& X_{0}=\partial_{u}, X_{1}=\partial_{r}, \quad X_{2}=\partial_{\phi}, \\
& X_{3}=\sin \phi \partial_{\theta}+\cot \theta \cos \phi \partial_{\phi}, \\
& X_{4}=-\cos \phi \partial_{\theta}+\cot \theta \sin \phi \partial_{\phi} .
\end{aligned}
$$

Out of these five NS, four are the basic KVs of the Kantowski-Sachs metric and $X_{0}$ is the symmetry corresponding to the Lagrangian. Here, $X_{0}$ represents the translation of geodesics parameter $u$ and $X_{1}$ is the translation of spatial coordinate $r$. The generators $X_{2}, X_{3}$ and $X_{4}$ form a Lie subalgebra for the rotation group $S O(3)$. The values of the Kantowski-Sachs metric functions for which the corresponding Lagrangian admit the above minimal NS are given in the following Table 1: 
Table 1. Exact form of metrics admitting five Noether symmetries (NS).

\begin{tabular}{ccc}
\hline No. & $\lambda(t)$ & $v(t)$ \\
\hline 3a & $\lambda^{\prime} \neq 0$ & $v^{\prime \prime} \neq 0$ and $v \neq \cosh t$ \\
3b & $\lambda \neq \sinh t$ & $v=\cosh t$ \\
3c & $\left(\lambda \lambda^{\prime}\right)^{\prime \prime} \neq 0$ & $v=$ const. $=\xi$ \\
3d & $\lambda=\sqrt{a t^{2}+2 b t+c} ; a \neq 0$, & $v=$ const. $=\xi$ \\
3e & $\lambda=\sqrt{2 b t+c}$ & $v=$ const. $=\xi$ \\
\hline
\end{tabular}

The corresponding Lie algebra for generators given in Equation (24) is:

$$
\left[X_{3}, X_{2}\right]=X_{4},\left[X_{2}, X_{4}\right]=X_{3},\left[X_{4}, X_{3}\right]=X_{2}, \quad\left[X_{i}, X_{j}\right]=0 \text {, otherwise. }
$$

Using Equation (8), the conserved forms of these NS become:

$$
\begin{aligned}
& I_{0}=-\dot{t}^{2}+\lambda^{2}(t) \dot{r}^{2}+v^{2}(t) \dot{\theta}^{2}+v^{2}(t) \sin ^{2} \theta \dot{\phi}^{2} \\
& I_{1}=-2 \lambda^{2}(t) \dot{r}^{2} \\
& I_{2}=-2 v^{2}(t) \sin ^{2} \theta \dot{\phi} \\
& I_{3}=-2 v^{2}(t)(\sin \phi \dot{\theta}+\cos \theta \cos \phi \sin \theta \dot{\phi}) \\
& I_{4}=-\frac{\partial I_{3}}{\partial \phi}
\end{aligned}
$$

The energy density and the Ricci scalar for Models $3 \mathrm{a}$ and $3 \mathrm{~b}$ are obtained as $T_{00}=\frac{1+v^{\prime 2}}{v^{2}}+\frac{2 \lambda^{\prime} v^{\prime}}{v \lambda}$ and $R=-\frac{2}{v^{2} \lambda}\left(2 v \lambda v^{\prime \prime}+v^{2} \lambda^{\prime \prime}+2 v v^{\prime} \lambda^{\prime}+\lambda v^{\prime 2}+\lambda\right)$. One can observe that the positive energy condition is satisfied if $\frac{\lambda^{\prime}}{\lambda}$ and $\frac{v^{\prime}}{v}$ have the same signs. Moreover, the Ricci scalar remains non-singular for $t \rightarrow 0$.

For the remaining three models, the energy density and the Ricci scalar are given by:

$$
T_{00}=\frac{1}{\xi^{2}}, \quad R=-2\left(\frac{\lambda^{\prime \prime}}{\lambda}+\frac{1}{\xi^{2}}\right)
$$

such that the energy remains positive for any arbitrary non-zero $\xi$ and the Ricci scalar is regular for any function $\lambda$.

\section{Six Noether Symmetries}

The Lagrangian of the following Kantowski-Sachs metric admits six NS:

$$
d s^{2}=d t^{2}-\gamma^{2} d r^{2}-v^{2}(t)\left(d \theta^{2}+\sin ^{2} \theta d \phi^{2}\right),
$$

where $\gamma$ is a non-zero constant, $v^{\prime \prime} \neq 0$ and $v \neq \cosh t$. Out of these six NS, five are the same as given in Equation (24) and one extra Noether symmetry is given by:

$$
X_{5}=\frac{u}{\gamma^{2}} \partial_{r}, \quad F=-2 r
$$

The conserved form for this symmetry is $I_{5}=-2(u \dot{r}-r)$. The corresponding non-zero commutator is $\left[X_{0}, X_{5}\right]=\frac{1}{\gamma^{2}} X_{1}$.

The metric (27) is non-conformally flat, and its energy density is obtained as $T_{00}=\frac{1+v^{\prime 2}}{v^{2}}$, which is always positive. The Ricci scalar is given by $R=-\frac{2}{v^{2}}\left(1+v^{\prime 2}+2 v v^{\prime \prime}\right)$, which has no singularity at the origin.

\section{Seven Noether Symmetries}

There are seven metrics whose Lagrangian admit a seven-dimensional algebra of NS. Table 2 contains all these metrics along with the two extra NS, other than the minimal NS given in (24), their Lie algebra, and the corresponding conserved quantities. 
Table 2. Metrics admitting seven Noether symmetries.

\begin{tabular}{|c|c|c|c|c|c|}
\hline No. & $\lambda(t)$ & $v(t)$ & Noether Symmetries & Invariants & Lie Algebra \\
\hline $7(\mathrm{i})$ & $\sin (\beta t) ; \beta \neq 0$ & $\gamma \neq 0$ & $\begin{array}{l}X_{5}=\cosh (\beta r) \partial_{t}-\cot (\beta t) \sinh (\beta r) \partial_{r} \\
X_{6}=\sinh (\beta r) \partial_{t}-\cot (\beta t) \cosh (\beta r) \partial_{r}\end{array}$ & $\begin{array}{c}I_{5}=2 \cosh (\beta r) \dot{t}+2 \cos (\beta t) \sin (\beta t) \sinh (\beta r) \dot{r} \\
I_{6}=\frac{1}{\beta} \frac{\partial I_{5}}{\partial r}\end{array}$ & $\begin{array}{c}{\left[X_{1}, X_{5}\right]=\beta X_{6},\left[X_{1}, X_{6}\right]=\beta X_{5},} \\
{\left[X_{6}, X_{5}\right]=\beta X_{1} .}\end{array}$ \\
\hline $7(\mathrm{ii})$ & $\cos (\beta t)$ & $\gamma \neq 0$ & $\begin{array}{l}X_{5}=\cosh (\beta r) \partial_{t}+\tan (\beta t) \sinh (\beta r) \partial_{r} \\
X_{6}=\sinh (\beta r) \partial_{t}+\tan (\beta t) \cosh (\beta r) \partial_{r}\end{array}$ & $\begin{array}{c}I_{5}=2 \cosh (\beta r) \dot{t}-2 \cos (\beta t) \sin (\beta t) \sinh (\beta r) \dot{r} \\
I_{6}=\frac{1}{\beta} \frac{\partial I_{5}}{\partial r}\end{array}$ & Same as in case 7(i) \\
\hline 7(iii) & $\sinh (\beta t) ; \beta \neq 0$ & $\gamma \neq 0$ & $\begin{array}{l}X_{5}=\cosh (\beta r) \partial_{t}-\operatorname{coth}(\beta t) \sinh (\beta r) \partial_{r} \\
X_{6}=\sinh (\beta r) \partial_{t}-\operatorname{coth}(\beta t) \cosh (\beta r) \partial_{r}\end{array}$ & $\begin{array}{c}I_{5}=2 \cosh (\beta r) \dot{t}+2 \cosh (\beta t) \sinh (\beta t) \sinh (\beta r) \dot{r} \\
I_{6}=\frac{1}{k} \frac{\partial I_{5}}{\partial r}\end{array}$ & Same as in case $7(\mathrm{i})$ \\
\hline 7 (iv) & $\cosh (\beta t)$ & $\gamma \neq 0$ & $\begin{array}{l}X_{5}=\cos (\beta r) \partial_{t}-\tanh (\beta t) \sin (\beta r) \partial_{r} \\
X_{6}=\sin (\beta r) \partial_{t}+\tanh (\beta t) \cos (\beta r) \partial_{r}\end{array}$ & $\begin{array}{c}I_{5}=2 \cos (\beta r) \dot{t}+2 \cosh (\beta t) \sinh (\beta t) \sin (\beta r) \dot{r} \\
I_{6}=\frac{1}{\beta} \frac{\partial I_{5}}{\partial r}\end{array}$ & Same as in case $7(\mathrm{i})$ \\
\hline $7(v)$ & $e^{\beta t}$ & $\gamma \neq 0$ & $\begin{array}{c}X_{5}=\partial_{t}-\beta r \partial_{r} \\
X_{6}=r \partial_{t}-\frac{e^{-2 \beta t}}{2 \beta} \partial_{r}-\frac{\beta r^{2}}{2} \partial_{r}\end{array}$ & $\begin{array}{c}I_{5}=2 \dot{t}+2 \beta r e^{2 \beta t} \dot{r} \\
I_{6}=2 r \dot{t}+\frac{\dot{r}}{\beta}+\beta r^{2} e^{2 \beta t} \dot{r}\end{array}$ & $\begin{array}{c}{\left[X_{5}, X_{1}\right]=\beta X_{1},\left[X_{1}, X_{6}\right]=X_{5}} \\
{\left[X_{6}, X_{5}\right]=\beta X_{6}}\end{array}$ \\
\hline $7(\mathrm{vi})$ & $c_{1} t+c_{2}$ & $v=\lambda$ & $\begin{array}{c}X_{5}=\frac{u^{2}}{2} \partial_{s}+\frac{u\left(c_{1} t+c_{2}\right)}{2 c_{1}} \partial_{t} ; F=\frac{t\left(c_{1} t+2 c_{2}\right)}{2 c_{1}} \\
V_{6}=u \partial_{u}+\frac{c_{1} t+c_{2}}{2 c_{1}} \partial_{t}\end{array}$ & $\begin{array}{c}I_{5}=\frac{u^{2}}{2} L+\frac{u\left(c_{1} t+c_{2}\right) \dot{t}}{c_{1}}-\frac{t^{2}}{2}-\frac{c_{2} t}{c_{1}} \\
I_{6}=u L+\frac{\left(c_{1} t+c_{2}\right) \dot{t}}{c_{1}}\end{array}$ & $\begin{array}{c}{\left[X_{0}, X_{5}\right]=X_{6},\left[X_{0}, X_{6}\right]=X_{0}} \\
{\left[X_{6}, X_{5}\right]=X_{5}}\end{array}$ \\
\hline 7 (vii) & $\begin{array}{c}\left(c_{1} t+c_{2}\right)^{\frac{\alpha-2 \beta}{\alpha}} \\
\alpha \neq 0\end{array}$ & $c_{1} t+c_{2}$ & $\begin{array}{c}X_{5}=u \partial_{u}+\frac{c_{1} t+c_{2}}{2 c_{1}} \partial_{t} \\
X_{6}=r \partial_{r}\end{array}$ & $\begin{array}{c}I_{5}=u L+\frac{\left(c_{1} t+c_{2}\right) \dot{t}}{c_{1}} \\
I_{6}=-2 r\left(c_{1} t+c_{2}\right)^{\frac{2(\alpha-2 \beta)}{\alpha} \dot{r}}\end{array}$ & $\begin{array}{c}{\left[X_{0}, X_{5}\right]=X_{0},\left[X_{1}, X_{6}\right]=X_{1}} \\
{\left[X_{6}, X_{1}\right]=X_{5}}\end{array}$ \\
\hline
\end{tabular}


The metric 7(i) is non-conformally flat, whose NS other than the minimal set of NS are KVs. Here, the generators $X_{1}, X_{5}$ and $X_{6}$ form a Lie algebra for the rotation group $S O(3)$.

Similarly, the metric 7(ii) is non-conformally flat, and it admits seven NS, five of which are the same as given in (24), and the remaining two are KVs, represented by $X_{5}$ and $X_{6}$.

The energy density and the Ricci scalar for the models 7(i) and 7(ii) are obtained as $T_{00}=\frac{1}{\gamma^{2}}$ and $R=2\left(\beta^{2}-\frac{1}{\gamma^{2}}\right)$. The positive energy condition clearly holds, and the spacetimes remain regular at the origin, as the Ricci scalar is constant.

The non-conformally-flat metric 7(iii) admits five NS as given in (24) along with two extra KVs, which are represented by $X_{5}$ and $X_{6}$.

The Weyl tensor for the metric 7(iv) has non-zero components, and hence, it is non-conformally flat. This metric possesses seven NS, out of which five are the same as given in (24) and the extra two are KVs.

The metric $7(\mathrm{v})$ is non-conformally flat, which admits two extra NS $X_{5}$ and $X_{6}$, which are KVs.

The energy density $T_{00}$ and the Ricci scalar $R$ for the models 7(iii), 7(iv), and 7(v) are:

$$
T_{00}=\frac{1}{\gamma^{2}}, \quad R=-2\left(\beta^{2}+\frac{1}{\gamma^{2}}\right),
$$

such that the positive energy condition is satisfied and the Ricci scalar is non-singular at the origin.

There exist two extra NS other than the minimal set of five NS for the non-conformally-flat metric $7\left(\right.$ vi). The Noether symmetry $X_{6}$ corresponds to a homothetic vector $\frac{c_{1} t+c_{2}}{2 c_{1}} \partial_{t}$, with the homothetic constant $\psi=\frac{1}{2}$. For this model, the energy density and the Ricci scalar become:

$$
T_{00}=\frac{1+3 c_{1}^{2}}{\left(c_{1} t+c_{2}\right)^{2}}, \quad R=-\frac{2\left(3 c_{1}^{2}+1\right)}{\left(c_{1} t+c_{2}\right)^{2}} .
$$

For any non-zero values of $c_{1}$ and $c_{2}$, the energy density is positive and the Ricci scalar remains non-singular at $t \rightarrow 0$.

Finally, the metric 7 (vii) is non-conformally flat, which admits the minimal set of five NS along with two extra NS $X_{5}$ and $X_{6}$. Here, $X_{6}$ is a KV, and $X_{5}$ corresponds to a homothetic vector $\frac{c_{1} t+c_{2}}{2 c_{1}} \partial_{t}$, with the homothetic constant $\psi=\frac{1}{2}$. The energy density and Ricci scalar for this metric are given as:

$$
T_{00}=\frac{\alpha+3 c_{1}^{2} \alpha-4 c_{1}^{2} \beta}{\alpha\left(c_{1} t+c_{2}\right)^{2}}, \quad R=-\frac{2\left(3 c_{1}^{2} \alpha^{2}-6 c_{1}^{2} \alpha \beta+4 c_{1}^{2} \beta^{2}+\alpha^{2}\right)}{\alpha^{2}\left(c_{1} t+c_{2}\right)^{2}} .
$$

For $c_{2} \neq 0$, the Ricci scalar has no singularity when $t \rightarrow 0$. The energy density may vary for different values of the constants involved in $T_{00}$. The following two graphs in Figure 1 show positive and negative energy density for some particular choices of the values of these constants.

As far as the case in which the positive energy condition is not met, it could represent an accelerated phase of expansion that may possibly be attributed to the dark energy. 


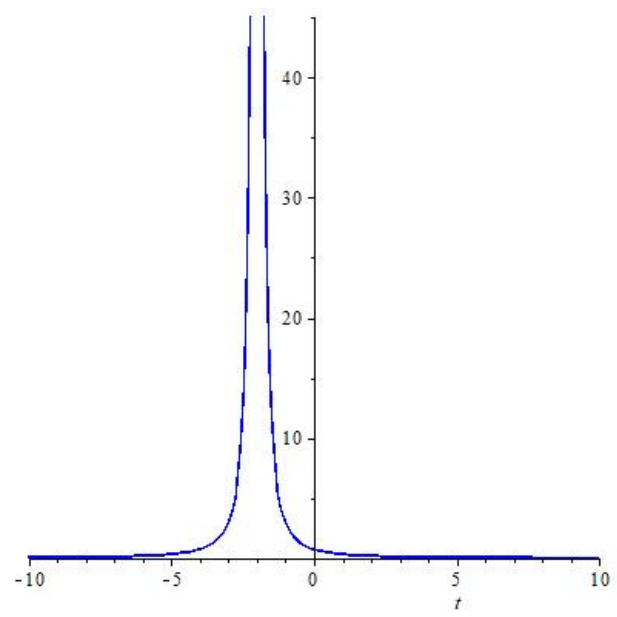

(a) $\alpha=\beta, c_{1}=0.5$ and $c_{2}=1$

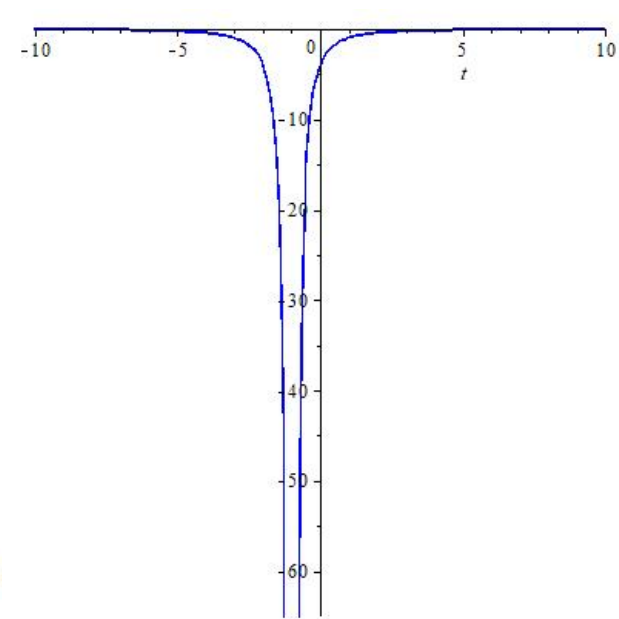

(b) $c_{1}=\alpha=c_{2}=1$ and $\beta=2$

Figure 1. Graphs of $T_{00}$.

\section{Eight Noether Symmetries}

The following is only one Kantowski-Sachs metric whose Lagrangian admits eight NS:

$$
d s^{2}=d t^{2}-\gamma^{2} d r^{2}-(\alpha t+\beta)^{2}\left(d \theta^{2}+\sin ^{2} \theta d \phi^{2}\right)
$$

where $\alpha \neq 0$ and $\gamma \neq 0$. The Weyl tensor for the above metric has non-vanishing components, and it is non-conformally flat. The set of eight NS for this metric contains the set of minimal five NS, given in (24), and the remaining three symmetries are:

$$
\begin{aligned}
& X_{5}=\frac{u^{2}}{2} \partial_{u}+\frac{u(\alpha t+\beta)}{2 \alpha} \partial_{t}+\frac{u r}{2} \partial_{r} ; F=\frac{t^{2}}{2}+\frac{\beta t}{\alpha}-\frac{r^{2} \gamma^{2}}{2}, \\
& X_{6}=\frac{u}{\gamma^{2}} \partial_{r} ; F=-2 r \\
& X_{7}=u \partial_{u}+\frac{\alpha t+\beta}{2 \alpha} \partial_{t}+\frac{r}{2} \partial_{r} .
\end{aligned}
$$

Out of the above three NS, $X_{7}$ corresponds to a homothetic vector, given by $\frac{\alpha t+\beta}{2 \alpha} \partial_{t}+\frac{r}{2} \partial_{r}$. The conserved form for these symmetries and Lie algebra are listed below:

$$
\begin{gathered}
I_{5}=\frac{u^{2}}{2}\left(-\dot{t}^{2}+\gamma^{2} \dot{r}^{2}+(\alpha t+\beta)^{2}\left(\dot{\theta}^{2}+\sin ^{2} \theta \dot{\phi}^{2}\right)\right)+\frac{u(\alpha t+\beta) \dot{t}}{\alpha}-u r \gamma^{2} \dot{r}-\frac{t^{2}}{2}-\frac{\beta t}{\alpha}+\frac{r^{2} \gamma^{2}}{2}, \\
I_{6}=-2 u \dot{r}+2 r \\
I_{7}=u\left(-\dot{t}^{2}+\gamma^{2} \dot{r}^{2}+(\alpha t+\beta)^{2}\left(\dot{\theta}^{2}+\sin ^{2} \theta \dot{\phi}^{2}\right)\right)+\frac{(\alpha t+\beta) \dot{t}}{\alpha}-r \gamma^{2} \dot{r} . \\
{\left[X_{0}, X_{5}\right]=X_{7},\left[X_{0}, X_{7}\right]=X_{0},\left[X_{0}, X_{6}\right]=\frac{1}{\gamma^{2}} X_{1},\left[X_{1}, X_{5}\right]=\frac{\gamma^{2}}{2} X_{6},} \\
{\left[X_{1}, X_{7}\right]=\frac{1}{2} X_{1},\left[X_{7}, X_{5}\right]=X_{7},\left[X_{7}, X_{6}\right]=\frac{1}{2} X_{6} .}
\end{gathered}
$$

The energy density and the Ricci scalar for Model (31) are respectively given by $T_{00}=\frac{1+\alpha^{2}}{(\alpha t+\beta)^{2}}$ and $R=-\frac{2}{(\alpha t+\beta)^{2}}$. Here, the positive energy condition holds, and for $t \rightarrow 0$, the scalar $R$ remains regular if $\beta \neq 0$. 


\section{Nine Noether Symmetries}

Here, we present some Kantowski-Sachs metrics, whose Lagrangian admits nine NS.

$$
\text { (1). } \quad d s^{2}=d t^{2}-\beta^{2} d r^{2}-\cosh ^{2} t\left(d \theta^{2}+\sin ^{2} \theta d \phi^{2}\right),
$$

where $\beta \neq 0$. The Weyl tensor for the above metric vanishes, and hence, it is conformally flat. The set of NS for this metric contains the minimal set of five symmetries given in (24), and four extra NS are admitted, which are listed below:

$$
\begin{aligned}
& X_{5}=u \partial_{r} ; \quad F=-2 r, \\
& X_{6}=\sin \theta \sin \phi \partial_{t}+\tanh t \cos \theta \sin \phi \partial_{\theta}+\csc \theta \tanh t \cos \phi \partial_{\phi}, \\
& X_{7}=-\sin \theta \cos \phi \partial_{t}-\tanh t \cos \theta \cos \phi \partial_{\theta}+\csc \theta \tanh t \sin \phi \partial_{\phi}, \\
& X_{8}=-\cos \theta \partial_{t}+\tanh t \sin \theta \partial_{\theta} .
\end{aligned}
$$

Here, $X_{6}, X_{7}$, and $X_{8}$ are KVs, while $X_{5}$ is a non-trivial Noether symmetry. The Lie algebra in this case gives:

$$
\begin{gathered}
{\left[X_{0}, X_{5}\right]=X_{1},\left[X_{6}, X_{2}\right]=\left[X_{4}, X_{8}\right]=X_{7},\left[X_{2}, X_{7}\right]=\left[X_{3}, X_{8}\right]=X_{6},} \\
{\left[X_{6}, X_{3}\right]=\left[X_{7}, X_{4}\right]=X_{8},\left[X_{6}, X_{7}\right]=X_{2},\left[X_{6}, X_{8}\right]=X_{3},\left[X_{7}, X_{8}\right]=X_{4},}
\end{gathered}
$$

and the conservation laws are:

$$
\begin{aligned}
& I_{5}=-2\left(u \beta^{2} \dot{r}-r\right), \\
& I_{6}=2 \sin \theta \sin \phi \dot{t}-2 \sinh t \cosh t(\cos \theta \sin \phi \dot{\theta}+\sin \theta \cos \phi \dot{\phi}), \\
& I_{7}=-2 \sin \theta \cos \phi \dot{t}+2 \sinh t \cosh t(\cos \theta \cos \phi \dot{\theta}-\sin \theta \sin \phi \dot{\phi}), \\
& I_{8}=-2 \cos \theta \dot{t}-2 \sinh t \cosh t \sin \theta \dot{\theta} .
\end{aligned}
$$

The energy density and Ricci scalar for the metric (34) are respectively given by $T_{00}=1$ and $R=-6$, such that the positive energy condition is satisfied and $R$ is regular at the origin.

$$
\text { (2). } d s^{2}=d t^{2}-\alpha^{2} d r^{2}-\beta^{2}\left(d \theta^{2}+\sin ^{2} \theta d \phi^{2}\right),
$$

where $\alpha \neq 0$ and $\beta \neq 0$. For the above metric, the Weyl tensor has non-zero components. The extra four NS other than the minimal set of NS, given in (24), for this metric are:

$$
\begin{aligned}
& X_{5}=\partial_{t}, \\
& X_{6}=-u \partial_{t} ; F=-2 t, \\
& X_{7}=\frac{u}{\alpha^{2}} \partial_{r} ; F=-2 r, \\
& X_{8}=r \partial_{t}+\frac{t}{\alpha^{2}} \partial_{r} .
\end{aligned}
$$

Here, $X_{5}$ and $X_{8}$ are KVs, while $X_{6}$ and $X_{7}$ are non-trivial NS. The Lie algebra and the conserved quantities are given as:

$$
\begin{aligned}
{\left[X_{6}, X_{0}\right]=} & {\left[X_{8}, X_{1}\right]=X_{5}, \quad\left[X_{0}, X_{7}\right]=\left[X_{5}, X_{8}\right]=\frac{1}{\alpha^{2}} X_{1}, } \\
& {\left[X_{8}, X_{6}\right]=X_{7}, \quad\left[X_{8}, X_{7}\right]=\frac{1}{\alpha^{2}} X_{6} . } \\
I_{5}= & 2 \dot{t}, \quad I_{6}=-2 u \dot{t}+2 t, \quad I_{7}=-2 u \dot{r}+2 r \\
I_{8}= & 2 r \dot{t}-2 t \dot{r} .
\end{aligned}
$$

The corresponding $T_{00}$ and $R$ for the above metric are given by $T_{00}=\frac{1}{\beta^{2}}$ and $R=-\frac{2}{\beta^{2}}$. Clearly, the energy density is positive for any value of $\beta$, and the Ricci scalar is regular at the origin. 


$$
\text { (3). } d s^{2}=d t^{2}-(\alpha t+\beta)^{2} d r^{2}-\xi^{2}\left(d \theta^{2}+\sin ^{2} \theta d \phi^{2}\right),
$$

where $\xi \neq 0$. The above metric is non-conformally flat, and it admits the following four NS along with five minimal symmetries, given in Equation (24).

$$
\begin{aligned}
& X_{5}=-u \cosh (\alpha r) \partial_{t}+\frac{u}{\alpha t+\beta} \sinh (\alpha r) \partial_{r} ; \quad F=-\frac{2 \beta}{\alpha} \cosh (\alpha r), \\
& X_{6}=-u \sinh (\alpha r) \partial_{t}+\frac{u}{\alpha t+\beta} \cosh (\alpha r) \partial_{r} ; \quad F=-\frac{2 \beta}{\alpha} \sinh (\alpha r), \\
& X_{7}=\cosh (\alpha r) \partial_{t}-\frac{\sinh (\alpha r)}{\alpha t+\beta} \partial_{r}, \\
& X_{8}=\sinh (\alpha r) \partial_{t}-\frac{\cosh (\alpha r)}{\alpha t+\beta} \partial_{r} .
\end{aligned}
$$

From the above, one can see that $X_{5}$ and $X_{6}$ are non-trivial NS, while $X_{7}$ and $X_{8}$ are KVs. In this case, the Lie algebra becomes:

$$
\begin{array}{ll}
{\left[X_{5}, X_{0}\right]=X_{7},} & {\left[X_{6}, X_{0}\right]=X_{8}, \quad\left[X_{1}, X_{5}\right]=\alpha X_{6},} \\
{\left[X_{1}, X_{6}\right]=\alpha X_{5}, \quad\left[X_{1}, X_{7}\right]=\alpha X_{8}, \quad\left[X_{1}, X_{8}\right]=\alpha X_{7} .}
\end{array}
$$

The conserved forms of the symmetries are given as follows:

$$
\begin{aligned}
& I_{5}=-2 u(\cosh (\alpha r) \dot{t}+(\alpha t+\beta) \sinh (\alpha r) \dot{r})+\frac{2 \beta}{\alpha} \cosh (\alpha r), \\
& I_{6}=\frac{1}{\alpha} \frac{\partial I_{5}}{\partial r} \\
& I_{7}=2(\cosh (\alpha r) \dot{t}+(\alpha t+\beta) \sinh (\alpha r) \dot{r}), \\
& I_{8}=\frac{1}{\alpha} \frac{\partial I_{7}}{\partial r} .
\end{aligned}
$$

For the above model, we have $T_{00}=\frac{1}{\xi^{2}}$ and $R=-\frac{2}{\xi^{2}}$ such that the energy density is positive and the Ricci scalar has no singularity at the origin.

\section{Eleven Noether Symmetries}

For $\lambda=\sinh t$ and $v=\cosh t$, we have the following Kantowski-Sachs metric, whose Lagrangian admits eleven NS:

$$
d s^{2}=d t^{2}-\sinh ^{2} t d r^{2}-\cosh ^{2} t\left(d \theta^{2}+\sin ^{2} \theta d \phi^{2}\right) .
$$

This metric has a zero Weyl tensor, and hence, it is conformally flat. The energy density and the Ricci scalar for the above model are obtained as $T_{00}=3$ and $R=-12$. Here, the positive energy condition is clearly satisfied, and $R$ is non-singular at the origin. The extra six NS (KVs) for the above metric are as follows:

$$
\begin{aligned}
X_{5} & =\sin \theta \sin \phi\left(\sinh r \partial_{t}-\operatorname{coth} t \cosh r \partial_{r}\right)+\tanh t \sinh r\left(\cos \theta \sin \phi \partial_{\theta}+\csc \theta \cos \phi \partial_{\phi}\right), \\
X_{6} & =\operatorname{same~as~} X_{5} \text { with } \sinh r \leftrightarrow \cosh r \\
X_{7} & =-\sin \theta \cos \phi\left(\sinh r \partial_{t}-\operatorname{coth} t \cosh r \partial_{r}\right)-\tanh t \sinh r\left(\cos \theta \cos \phi \partial_{\theta}-\csc \theta \sin \phi \partial_{\phi}\right), \\
X_{8} & =\operatorname{same~as~} X_{7} \text { with } \sinh r \leftrightarrow \cosh r \\
X_{9} & =-\cos \theta\left(\sinh r \partial_{t}-\operatorname{coth} t \cosh r \partial_{r}\right)+\tanh t \sinh r \sin \theta \partial_{\theta}, \\
X_{10} & =\text { same as } X_{9} \text { with } \sinh r \leftrightarrow \cosh r
\end{aligned}
$$

One may easily simplify Equation (8) to find the expressions for the corresponding conservation laws for the above generators. The Lie algebra in this case is obtained as: 


$\begin{array}{lllll}{\left[X_{1}, X_{5}\right]=X_{6,}} & {\left[X_{1}, X_{6}\right]=X_{5},} & {\left[X_{1}, X_{7}\right]=X_{8},} & {\left[X_{1}, X_{8}\right]=X_{7},} & {\left[X_{1}, X_{9}\right]=X_{10}} \\ {\left[X_{1}, X_{10}\right]=X_{9},} & {\left[X_{5}, X_{2}\right]=X_{7},} & {\left[X_{6}, X_{2}\right]=X_{8},} & {\left[X_{2}, X_{7}\right]=X_{5},} & {\left[X_{2}, X_{8}\right]=X_{6}} \\ {\left[X_{5}, X_{3}\right]=X_{9},} & {\left[X_{6}, X_{3}\right]=X_{10},} & {\left[X_{3}, X_{9}\right]=X_{5},} & {\left[X_{3}, X_{10}\right]=X_{6},} & {\left[X_{7}, X_{4}\right]=X_{9}} \\ {\left[X_{8}, X_{4}\right]=X_{10},} & {\left[X_{4}, X_{9}\right]=X_{7},} & {\left[X_{4}, X_{10}\right]=X_{8},} & {\left[X_{5}, X_{6}\right]=X_{1},} & {\left[X_{7}, X_{5}\right]=X_{2}} \\ {\left[X_{9}, X_{5}\right]=X_{3},} & {\left[X_{6}, X_{8}\right]=X_{2},} & {\left[X_{6}, X_{10}\right]=X_{3},} & {\left[X_{7}, X_{8}\right]=X_{1},} & {\left[X_{9}, X_{7}\right]=X_{4}} \\ {\left[X_{8}, X_{10}\right]=X_{4},} & {\left[X_{9}, X_{10}\right]=X_{1},} & & & \end{array}$

\section{Summary and Discussion}

In this paper, we have classified the Lagrangian of the Kantowski-Sachs metric via its NS. The set of determining equations is obtained and then integrated in several cases. It is observed that the Kantowski-Sachs metric admits a 5-, 6-, 7-, 8-, 9-, or 11-dimensional Lie algebra of NS for different values of the metric functions. The number of non-trivial NS for this metric is shown to be one, two, or three, while the number of KVs is found to be 4,5,6,7, or 10 .

We have found five different metrics, each admitting the minimal set of five NS, out of which four are the minimum KVs of the Kantowski-Sachs metric and one is a non-trivial Noether symmetry, which is $\partial_{u}$. The gauge function is trivial here.

There is only one metric (27) that admits six NS. This set of six NS contains the minimal set of five NS along with one extra Noether symmetry with the gauge function $F=-2 r$.

In the case of seven-dimensional Noether algebra, we have obtained seven different metrics. For the first five metrics, 7(i)-7(v), we have six KVs and one Noether symmetry $\partial_{u}$, with a trivial gauge function. For metric $7(\mathbf{v i})$, we have the minimal set of NS along with two extra NS, given by $X_{5}$ and $X_{6}$. The gauge function corresponding to $X_{5}$ is found to be $F=\frac{t\left(c_{1} t+2 c_{2}\right)}{2 c_{1}}$. It can bee seen that the Noether symmetry $X_{6}$ for this metric corresponds to an $\operatorname{HV} \frac{c_{1} t+c_{2}}{2 c_{1}} \partial_{t}$, with homothetic constant $\psi=\frac{1}{2}$. Similar results are obtained for the metric $\mathbf{7 ( v i i ) . ~ H e r e , ~ t h e ~ n u m b e r ~ o f ~ K V s ~ i s ~ f i v e . ~}$

There exists only one metric (31) possessing eight NS, of which five are the minimal NS for the Kantowski-Sachs metric, and three extra NS are obtained, which are presented in (32). One of these three NS, denoted as $X_{7}$, corresponds to an $\mathrm{HV} \frac{\alpha t+\beta}{2 \alpha} \partial_{t}+\frac{r}{2} \partial_{r}$. The number of KVs for this metric is only four.

In the case of the nine-dimensional Lie algebra of NS, we have found three metrics (34), (37), and (40). For the metric (34), we have the minimal set of five NS along with four extra symmetries, of which three are KVs and one is a non-trivial Noether symmetry $u \partial_{r}$ with the gauge function $F=-2 r$. The number of KVs in this case is seven. For the remaining two metrics (37) and (40), we have six KVs along with three non-trivial NS.

Finally, we have only one metric (43) where the dimension of the algebra of NS is 11. Out of eleven NS, ten are the KVs, and there is only one non-trivial Noether symmetry, given by $\partial_{u}$.

For almost all the obtained metrics, it is observed that the positive energy condition is satisfied, and the corresponding Ricci scalar has no singularity at the origin.

Author Contributions: This work is an original research work prepared by S.S.A. under the joint mentorship of T.H. and A.H.B.

Funding: This research received no external funding.

Acknowledgments: We are thankful to the referees for their invaluable suggestions due to which the manuscript was significantly improved. Sumaira Saleem Akhtar also acknowledges the Higher Education Commission of Pakistan for granting the Indigenous fellowship for her Ph.D.

Conflicts of Interest: The authors declare no conflict of interest.

\section{References}

1. Stephani, H.; Kramer, D.; Maccallum, M.; Hoenselaers, C.; Herlt, E. Exact Solutions of Einstein's Field Equations, 2nd ed.; Cambridge University Press: Cambridge, UK, 2003.

2. Meisner, C.W.; Thorne, K.S.; Wheeler, J.A. Gravitation; Benjamin: New York, NY, USA, 1973. 
3. Petrov, A.Z. Einstein Spaces; Pergamon: Oxford, UK, 1969.

4. Bokhari, A.H.; Kashif, A.R.; Qadir, A. Classification of curvature collineations of plane symmetric static spacetimes. J. Math. Phys. 2000, 41, 2167-2172. [CrossRef]

5. Hall, G.S.; da Costa, J. Curvature collineations in general relativity. I. J. Math. Phys. 1991, 32, $2848-2853$. [CrossRef]

6. Hall, G.S.; da Costa, J. Curvature collineations in general relativity. II. J. Math. Phys. 1991, 32, $2854-2862$. [CrossRef]

7. Hussain, T.; Akhtar, S.S.; Khan, S. Ricci inheritance collineation in Bianchi type I spacetimes. Eur. Phys. J. Plus 2015, 130, 44. [CrossRef]

8. Hussain, T.; Akhtar, S.S.; Bokhari, A.H.; Khan, S. Ricci inheritance collineations in Bianchi type II spacetime. Mod. Phys. Lett. A 2016, 31, 1650102. [CrossRef]

9. Akhtar, S.S.; Hussain, T.; Bokhari, A.H.; Khan, F. Conformal collineations of the Ricci and energy-momentum tensors in static plane symmetric space-times. Theor. Math. Phys. 2018, 195, 595-601. [CrossRef]

10. Noether, E. Invariant variations problems. Nachr. Konig. Gissell. Wissen. Got-tingen Math.-Phys. Kl. 1918, 2, 235-257; English translation in Transp. Theory Stat. Phys. 1971, 1, 186-207. [CrossRef]

11. Hickman, M.; Yazdan, S. Noether symmetries of Bianchi type II spacetimes. Gen. Relativ. Gravit. 2017, 49, 65.

12. Bokhari, A.H.; Kara, A.H. Noether versus Killing symmetry of conformally flat Friedmann metric. Gen. Relativ. Gravit. 2007, 39, 2053-2059. [CrossRef]

13. Bokhari, A.H.; Kara, A.H.; Kashif, A.R.; Zaman, F.D. Noether symmetries versus Killing vectors and isometries of spacetimes. Int. J. Theor. Phys. 2006, 45, 1029-1039. [CrossRef]

14. Camci, U. Symmetries of geodesic motion in Gödel-type spacetimes. J. Cosmol. Astropart. Phys. 2014, 2014. [CrossRef]

15. Camci, U.; Yildirim, A. Noether gauge symmetry classes for pp-wave spacetimes. Int. J. Geom. Meth. Mod. Phys. 2015, 12, 1550120. [CrossRef]

16. Ali, F.; Feroze, T. Classification of Plane Symmetric Static Space-Times According to Their Noether Symmetries. Int. J. Theor. Phys. 2013, 52, 3329-3342. [CrossRef]

17. Ali, F.; Feroze, T.; Ali, S. Complete classification of spherically symmetric static spacetimes via Noether symmetries. Theor. Math. Phys. 2015, 184, 973-985. [CrossRef]

18. Ali, F.; Feroze, T. Complete classification of cylindrically symmetric static spacetimes and the corresponding conservation laws. Sigma Math. 2016, 4, 50. [CrossRef]

19. Jamil, B.; Feroze, T. Conservation laws corresponding to the Noether symmetries of the geodetic Lagrangian in spherically symmetric spacetimes. Int. J. Mod. Phys. D 2017, 26. [CrossRef]

20. Jamil, B.; Feroze, T.; Vargas, A. Geometrical/Physical interpretation of the conserved quantities corresponding to Noether symmetries of plane symmetric spacetimes. Adv. Math. Phys. 2017, 2017, 4384093. [CrossRef]

21. Paliathanasis, A.; Tsamparlis, M.; Mustafa, M.T. Symmetry analysis of the Klein-Gordon equation in Bianchi I spacetimes. Int. J. Geom. Meth. Mod. Phys. 2015, 12, 1550033. [CrossRef]

22. Tsamparlis, M.; Paliathanasis, A. The geometric nature of Lie and Noether symmetries. Gen. Relativ. Gravit. 2011, 43, 1861-1881. [CrossRef]

23. Ali, S.; Hussain, I. A study of positive energy condition in Bianchi V spacetimes via Noether symmetries. Eur. Phys. J. C 2016, 76. [CrossRef]

24. Usamah, S.A.; Bokhari, A.H.; Kara, A.H.; Shabbir, G. Classification of Variational Conservation Laws of General Plane Symmetric Spacetimes. Commun. Theor. Phys. 2017, 68, 335-341.

25. Ma, W.X. Conservation laws by symmetries and adjoint symmetries. Discret. Contin. Dyn. Syst. Ser. S 2018, 11,707-721. [CrossRef]

26. Ma, W.X.; Zhou, Y. Lump solutions to nonlinear partial differential equations via Hirota bilinear forms. J. Diff. Equ. 2018, 264, 2633-2659. [CrossRef]

27. Kantowski, R.; Sachs, R.K. Some Spatially Homogeneous Anisotropic Relativistic Cosmological Models. J. Math. Phys. 1966, 7, 443-446. [CrossRef]

28. Hand, L.N.; Finch, J.D. Analytical Mechanics; Cambridge University Press: Cambridge, UK, 2008.

(c) 2018 by the authors. Licensee MDPI, Basel, Switzerland. This article is an open access article distributed under the terms and conditions of the Creative Commons Attribution (CC BY) license (http:/ / creativecommons.org/licenses/by/4.0/). 